\title{
La boîte noire de l'intentionnalité animale
}

Notre propos part d'un constat : celui de l'ubiquité de la notion d'intentionnalité animale, pardelà les frontières disciplinaires, dans le champ scientifique contemporain. Le fait que les animaux puissent posséder des états mentaux plus ou moins complexes, et comparables dans une certaine mesure à des compétences cognitives identifiées chez l'humain, anime en effet autant les sciences du comportement (cognition sociale, écologie comportementale) que la philosophie de l'esprit, l'éthique ou la métaphysique - disciplines dans lesquelles l'intentionnalité animale est conceptualisée de manière fort diverse. D'une version très parcimonieuse en matière d'attribution d'états émotionnels ou représentationnels, à une version radicalement cognitiviste posant l'existence d'une théorie de l'esprit, les définitions de l'intentionnalité animale sont aussi nombreuses que ne le sont les manières d'éprouver empiriquement son existence.

L'interrogation n'est pas nouvelle, même si elle a pu se dissimuler derrière des termes plus généraux. Chez les philosophes antiques et classiques on trouve déjà les notions de « raison animale », ou d' " intelligence animale », en particulier dans les traités zoologiques $\mathrm{d}^{\prime}$ Aristote $^{1}$, mais aussi, de manière plus anecdotique, chez Montaigne (Livre 12, Tome 2), ou encore chez Bentham, pour lequel la sagesse accordée aux animaux repose sur le fait de les concevoir comme des êtres «sentients $»^{2}$. Dans les termes de la phénoménologie ou de la philosophie de l'esprit, il sera davantage question de «conscience animale $»^{3}$, ou encore d' " intentionnalité animale », termes qu'on retrouvera par ailleurs dans l'éthique animale ou l'écologie politique ${ }^{4}$. Psychologues, primatologues et neuroscientifiques s'intéresseront plutôt à l'idée d'une «théorie de l'esprit» pour caractériser des compétences spécifiques de certaines espèces ${ }^{5}$. La notion, née à la fin des années 1970 sous la plume des primatologues et psychologues David Premack et Guy Woodruff, est apparue pour la première fois dans un article devenu célèbre : " Does the chimpanzee have a theory of mind ? ». Elle a ensuite été abondamment reprise en primatologie ${ }^{6}$, et de manière intéressante, ressaisie par la philosophie de l'esprit pour examiner la spécificité du mode de raisonnement humain ${ }^{7}$. Toutes ces

\footnotetext{
${ }^{1}$ Voir Aristote, Génération des Animaux, trad. franç. L. Pierre. 2002, Paris, Les belles Lettres ; Aristote, Histoire des Animaux, trad. franç. J. Bertier, 1994, Paris, Gallimard; mais également Aristote, De l'âme, trad. franç. P. Thillet, 2005, Paris, Gallimard, et surtout Aristote, Éthique à Nicomaque, trad. franç. J. Tricot, 1990, Paris, Vrin, en particulier VI, 8, $1141 \mathrm{~b} 23$, note 5 .

${ }^{2}$ Jeremy Bentham, Introduction aux principes de morale et de législation. Analyse et philosophie, 2011 [1789], Paris, Vrin.

${ }^{3}$ Voir inter alia Colin Allen, « Animal Consciousness », In The Stanford Encyclopedia of Philosophy, édité par Edward N. Zalta. Disponible en ligne à l'adresse http://plato.stanford.edu/archives/win2011/entries/consciousness-animal/

${ }^{4}$ Voir récemment Pierre Le Neindre, Muriel Dunier, Raphaël Larrère, Patrick Prunet (Eds.) La conscience des animaux, 2018, Editions Quae.

${ }^{5}$ David Premack et Guy Woodruff, « Does the Chimpanzee Have a Theory of Mind? », Behavioral and Brain Sciences, 1978, vol 1 (4), p. 515-526.

${ }^{6}$ Voir Josep Call et Michael Tomasello, « Does the Chimpanzee Have a Theory of Mind? 30 Years Later », Trends in Cognitive Sciences, 1999, Vol.12 No.5.

${ }^{7}$ Voir par exemple dans la littérature anglo-saxone les travaux de Peter Carruthers: The Nature of the Mind: an Introduction, 2004, New York, London, Routledge ; ou encore «Meta-Cognition in Animals: a Skeptical Look », Mind and Language, 2008, 23, p. 58-89. En France, voir les travaux de la philosophe Joëlle Proust :
} 
occurrences (intelligence, raison, conscience, intentionnalité) interrogent à des degrés divers la capacité des animaux à forger des représentations mentales, et la mise en évidence de ces dernières sert des arguments très divers. Que ce soit donc pour l'interroger ${ }^{8}$, l'affirmer', la contester $^{10}$, ou encore la revendiquer pour justifier un meilleur traitement des bêtes d'élevage ${ }^{11}$, l'existence - et la mesure - d'une intentionnalité animale fait l'objet de constantes discussions. Mais si les termes ont évolué, le fond du débat n'a pas beaucoup changé depuis sa formulation cartésienne : quelle est la part mécaniciste ou d'instinct, quelle est la part occupée par l'esprit et les capacités de raisonnement, telles qu'elles se manifestent notamment dans la communication et la coopération?

La question de l'intentionnalité animale n'est toutefois pas seulement le fruit d'un vieux débat scolastique, constamment rejoué dans la sphère scientifique contemporaine. C'est aussi, et peut-être surtout, une question pratique qui se pose de manière cruciale aux individus et aux groupes qui vivent ordinairement au contact d'animaux, de manière quotidienne (propriétaires, vétérinaires, entraineurs) ou plus ponctuelle (promeneurs dans les parcs, visiteurs de zoo, etc.).

À partir de ce constat, l'idée générale de cet article est de proposer au lecteur d'envisager l'intentionnalité animale autrement que sous l'angle d'un problème de recherche auquel il s'agirait de répondre ( « les animaux possèdent-ils des compétences cognitives ? Font-ils des inférences? Sont-ils en capacité d'attribuer des états mentaux à autrui ? »). En adoptant une posture agnostique sur ces questions, il se distingue de la grande majorité de la production scientifique contemporaine. Je propose en effet ici plutôt de déployer une approche pragmatiste inspirée de l'ethnométhodologie, qui considère l'intentionnalité animale comme un objet théorique devant être respécifié, c'est-à-dire, en termes garfinkeliens (Garfinkel, 1967), rapporté à ses différents contextes d'usage.

Le principe, autrement dit, consiste à se déprendre des questions traditionnellement posées quand l'intentionnalité animale est soumise à un examen expérimental. Non pas qu'on ne croit pas que ces questions puissent avoir une certaine pertinence, dans bien des champs du savoir (pour les perspectives comparatistes notamment). Mais je suggère qu'il est sans doute au moins aussi heuristique de les aborder à partir des pratiques empiriques, et notamment à travers l'observation d'interactions en contexte naturel, et non expérimental. Les acteurs sociaux ordinaires ne sont pas des scientifiques, et pourtant ils apportent, dans leurs pratiques, des réponses de facto aux questions posées par ces derniers. Dans leurs interactions avec les animaux, dans les formes de raisonnement pratique $^{12}$ qu'ils mènent dans le cours de leurs

Comment l'esprit vient aux bêtes : essai sur la représentation, 1997, Paris, Gallimard ; ou Les animaux pensentils ?, 2003, Paris, Bayard.

${ }^{8}$ David Premack et Guy Woodruff, ibid.

${ }^{9}$ Donald Griffin, Animal Thinking, 1984, Cambridge, EU, Harvard University Press ; Joelle Proust, ibid.

10 Derek Penn et Daniel Povinelli, «On the Lack of Evidence that Non-HumanAanimals Possess Anything Remotely Resembling a 'Theory of Mind' », Philosophical Transactions of the Royal Society, 362, 2007, p. 731744 ; ou encore Michael Tomasello et Malinda Carpenter, "Shared intentionality » Special issue of Developmental Science, 10, 2007, p. 121-125.

11 Peter Singer, Animal Liberation: A New Ethics for our Treatment of Animals, 1975, New York Review/Random House ; Le Neindre et alii., ibid.

12 Pour une définition de la notion de raisonnement pratique en sociologie de l'action, voir Albert Ogien et Louis Quéré, Le vocabulaire de la sociologie de l'action, 2005, Paris, Ellipses, p. 99 : « le raisonnement pratique prend place entre le hasard et la routine (...) il intervient dès lors que la sérialité d'une conduite, la séquentialité d'un procès et l'intentionnalité d'une action sont organisées de façon intelligible et justifiable, soit avant, soit dans le 
actions, ils offrent des réponses pratiques à ces questions. Celles-ci, bien évidemment, ne se situent pas sur le même plan épistémique que celles apportées par la communauté scientifique. Ce sont des réponses pratiques en un sens aristotélicien : praxiques, orientées vers l'action. Il s'agit alors de se demander : Que font les individus et les groupes quand ils interprètent les actions animales à travers le prisme de l'intentionnalité ? Sur la base de quels motifs à l'inverse, la leur refusent-ils? Sur quelles ressources interactionnelles ou communicationnelles précises s'adossent leurs 'pratiques inférentielles' ? Comment, à partir de signes (au sens Peircien ${ }^{13}$ ) produits par les animaux, les acteurs sociaux parviennent-ils à générer des interprétations ? Comment un comportement somatique est-il retraduit en actions signifiantes ? Comment, en somme, parviennent-ils à donner un sens aux actions animales, de manière naturelle (i.e. sans forcément mobiliser de concepts ou de méthodes scientifiques, mais en s'appuyant sur une sorte d'herméneutique ordinaire, grâce à laquelle les comportements animaux sont rendus proprement compréhensibles, et partant, discutables et partageables)?

Le cheminement de cet article se déroulera donc en deux temps : d'abord je tâcherai de montrer quels sont les problèmes logiques inhérents à la manière dont une partie des sciences cognitives $^{14}$, en l'occurrence leurs courants les plus mentalistes, conçoivent et examinent empiriquement l'intentionnalité animale. J'examinerai en particulier la dimension circulaire sur laquelle s'appuie la démonstration, quand il s'agit de questionner l'existence d'une «théorie de l'esprit» chez l'animal. Dans un second temps, je tenterai de proposer une approche alternative, qui montre comment les individus et les groupes interagissant au contact d'animaux résolvent pratiquement ces questions dans leurs activités ordinaires. L'intentionnalité animale n'y est alors plus un problème scientifique, mais une ressource mobilisée à toutes fins pratiques pour les besoins de l'interaction.

\section{1) Les sophismes cognitivistes, ou l'existence d'une «boîte noire »}

Traitée comme une boîte noire, qu'il suffirait d'ouvrir (à condition toutefois de posséder les bonnes clés, c'est-à-dire des outils scientifiques suffisamment puissants), la notion d'intentionnalité animale essentialise un ensemble disparate de compétences cognitives et sociales. Une première étape du raisonnement sera donc consacrée à un retour sur les problèmes de logique posés par l'argument cognitiviste.

La notion de boîte noire est généralement plutôt associée à l'épistémologie behavioriste, pour laquelle le comportement résulte de la gestion d'un stimulus par l'organisme, sans que l'on cherche à savoir quels sont les processus qui le rendent possible. Dans un article intitulé « Who took the cog out of cognitive science? Mentalism in an era of anti-cognitivism », le

cours même de leur effectuation. Il est difficile de le décrire, du moins si l'on veut le saisir dans son effectivité pratique, c'est-à-dire selon ses modalités concrètes lorsqu'il est exercé en situation dans l'agir. On sait cependant identifier les marques de sa présence: là où une conduite non mécanique manifeste une cohérence, une pertinence, un ajustement ou un ordre justifiable, une forme ou une autre de raisonnement pratique est à l'œuvre ».

${ }^{13}$ Voir Chloé Mondémé « Une linguistique au-delà de l'humain ? Les vertus heuristiques de la sémiosis interespèce », à paraître en 2020, Revue Itinéraires [en ligne].

14 J'emploie le terme de « sciences cognitives » pour désigner ici la production mainstream en cognition sociale, étant cependant consciente qu'il existe une grande variété de courants au sein de la psychologie, notamment les perspectives enactivistes, la cognition distribuée ou située, qui adoptent des positions critiques vis-à-vis de l'approche « cognitiviste » dont il est question ici (voir notamment Ivan Leudar et Alan Costall, Against Theory of Mind, 2009, Palgrave MacMillan). 
psychologue Josef Perner revient sur cette conception, et sur ce que la révolution cognitive promettait : pouvoir enfin ouvrir cette boîte noire.

«Je me souviens du behaviorisme comme d'une doctrine de la boîte noire : tiens-t'en aux inputs et outputs observables, et abstiens-toi de théoriser sur la mécanique et les rouages internes du système qui créent les connexions causales entre les stimuli observables et les réponses. La révolution cognitive devait supposément ouvrir la boîte noire $»^{15}$.

Mais le développement rapide des sciences cognitives, et de toutes les disciplines de l'esprit qui lui sont connexes (neurosciences, cognition sociale), n'a manifestement pas rendu possible un accès transparent aux rouages de l'esprit. 40 ans après, l'une de ses questions emblématiques - est-ce que les grands singes sont capables de manifester une "théorie de l'esprit »? - reste amplement débattue, et ne fait toujours pas l'objet d'un consensus chez les primatologues et psychologues ${ }^{16}$.

Telle que formulée initialement, la « théorie de l'esprit» ou «ToM» (pour Theory of Mind) renvoie à la capacité qu'ont les individus d'imputer des états mentaux, à eux-mêmes ou à autrui. C'est une théorie au sens où (i) elle n'est pas directement observable dans les comportements, mais nécessite d'en passer par des relations d'inférences entre un comportement observé et un état mental imputé, et (ii) elle permet de faire des prédictions sur les comportements d'autrui ${ }^{17}$. L'idée, en somme, consiste à démontrer - ou réfuter l'existence d'une forme plus ou moins élaborée de cognition et d'intentionnalité chez les animaux. Mais de quoi parle-t-on ? De leur capacité à attribuer des états mentaux à leurs conspécifiques? À résoudre des problèmes de fausses croyances ? À avoir des dispositions pour des formes de métacognition? De manière significative, ce sont surtout les philosophes de ''esprit qui se sont ressaisis de cette notion $^{18}$, ce qui a permis de produire (là encore) des distinctions scalaires entre un stade de premier niveau (mindreading abilities), consistant en l'attribution de désirs et d'états épistémiques à autrui ${ }^{19}$, jusqu'à un stade plus élaboré, dont l'expérience révélatrice ultime serait la False Belief Task (tâche de la fausse croyance) ${ }^{20}$ nous y reviendrons.

Ces questions hautement spéculatives trouvent la plupart du temps leur retraduction empirique dans des protocoles expérimentaux très concrets. Et c'est là que le bât blesse. Deux critiques peuvent alors être formulées : l'une relative à un problème épistémologique (un problème de généralisation), l'autre à un problème logique (un raisonnement circulaire). La suite du développement reprend ces deux points.

Tout d'abord, il est frappant de voir que la plus grande part des tests visant à éprouver l'intelligence sociale des animaux, des grands singes en particulier, est directement héritée des protocoles mis au point en psychologie du développement. C'est le cas pour l'essentiel de la

\footnotetext{
${ }^{15}$ Josef Perner, «Who Took the Cog out of Cognitive Science? Mentalism in an Era of Anti-Cognitivism », in Peter Frensch et Ralph Scharzer (Eds.), International Perspectives on Psychological Science: Cognition and Neuropsychology,2010, New York, Taylor \& Francis, p. 241.

${ }^{16}$ Voir Josep Call et Michael Tomasello, idem.

${ }^{17}$ David Premack et Guy Woodruff, ibid., p. 515.

${ }^{18}$ Voir note 7 infra.

${ }^{19}$ Voir l'idée de «stage 1 mindreading abilities » développée notamment par Logan Fletcher et Peter Carruthers dans le chapitre "Behavior-Reading versus Mentalizing in Animals » in Janet Metcalfe et Herbert Terrace, Agency and Joint Attention, 2013, New York, Oxford University Press, p. 82-99.

${ }^{20}$ Ce test a été mis au point dans les années 1980 par deux psychologues autrichiens pour évaluer les capacités cognitives du jeune enfant: Wimmer Heinz et Josef Perner, "Beliefs about Beliefs: Representing and Constraining Function of Wrong Beliefs in Young Children's Understanding of Deception », Cognition, $\mathrm{n}^{\circ} 13$, 1983, p. 103-128.
} 
cognition sociale, qui s'adosse à la psychologie développementale humaine pour élaborer sa démarche comparatiste. En pratique, les protocoles des modèles humains sont appliqués chez les animaux. Or, le prix de cette transposition paraît couteux sur un plan épistémique. Reprenons le cas de la False Belief Task, à titre d'exemple. Dans ce test, on place les enfants face à une situation où un adulte dépose un morceau de chocolat ou un bonbon dans un placard, bleu par exemple. Un tiers (une poupée, un autre enfant) assiste à la scène. Puis le tiers se retire, et l'adulte déplace le chocolat pour le mettre dans un autre placard, vert par exemple. Quand le tiers revient, on demande à l'enfant où celui-ci va aller chercher le chocolat. La psychologie sociale met en évidence qu'avant 4 ans, les enfants projettent que le tiers se dirigera vers le placard vert (puisqu'après tout, c'est effectivement là qu'est le bonbon), alors qu'à 4 ans et plus, ils seront en mesure de projeter que le tiers se dirigera en priorité vers le placard bleu, c'est-à-dire là où était le chocolat quand il a quitté la pièce avant qu'il ne soit déplacé, et donc là où il a toutes les raisons de penser qu'il se trouve encore. Ce test a subi par la suite de nombreuses variantes ${ }^{21}$, mais le principe reste toujours le même : pour le réussir, un enfant doit être en mesure d'attribuer à autrui des croyances qui sont différentes des siennes propres (lui sait que le chocolat est dans le placard vert, mais la poupée ou l'autre enfant ne le sait pas). Comme on peut le voir, le test s'appuie sur un procédé complètement narratif, dont il n'est pas étonnant qu'il soit difficilement transposable à des formes de vie non-humaines. Comme la plupart des expériences en cognition sociale, il met littéralement "en scène » une situation relativement complexe, incluant des activités sociales (faire un gâteau, manger du chocolat), des allers et venues : en somme, un petit vaudeville qui pourrait être particulièrement difficile à saisir pour un chimpanzé captif. Il y a certes eu des tentatives pour concevoir des protocoles "non verbaux ${ }^{22}$ : sans grande surprise, ils ont conduit les scientifiques à conclure que les chimpanzés étaient incapables d'attribuer à autrui des états mentaux autres que les leurs propres ${ }^{23}$. Dans un article intitulé "The Mismeasure of Ape Social Cognition » (en référence à l'ouvrage de Steven J. Gould The Mismeasure of Man), les psychologues David Leavens, Kim Bart et William Hopkins ${ }^{24}$ produisent une critique remarquable des biais méthodologiques extrêmement problématiques qui apparaissent quand il s'agit de comparer les compétences sociales (ou l'intelligence sociale) des grands singes captifs et non acculturés à celles de jeunes enfants occidentaux, vivant dans des environnements sociaux très riches.

Dans tous les cas, le présupposé est lourd : le primate serait sur le plan phylogénétique ce que l'enfant est sur le plan ontogénétique : un précurseur de l'être rationnel et locuteur compétent qu'est l'adulte humain. Est-il sûr, cependant, que le bonobo de 2010, vivant dans telle réserve ou dans telle station de primatologie puisse être apparenté à 'l'ancêtre commun' qui le relie à

\footnotetext{
${ }^{21}$ Dans The Social Construction of What (1999), Ian Hacking ironise sur ce succès : « Une seule expérience ingénieuse, originellement suggérée par les philosophes, a engendré une industrie expérimentale. C'est souvent le cas en psychologie, où les nouvelles idées expérimentales sont aussi rares et difficiles à inventer que les preuves mathématiques ou les véritables tours de magie »» (je traduis, p. 115).

${ }^{22}$ Voir notamment Josep Call et Michael Tomasello, «A Non-Verbal False Belief Task: the Performance of Children and Great Apes », Child Development, 1999, vol 70 (2), p. 381-395; ou, dix ans plus tard, Carla Krachun, Melinda Carpenter, Josep Call et Michael Tomasello, 2009, « A Competitive Nonverbal False Belief Task for Children and Apes », Developmental Science, 2009 Jul;12(4), p. 521-535.

${ }^{23}$ Pour une critique interne aux sciences cognitives de l'usage de la False Belief Task comme test pertinent pour la mise en évidence de la ToM, voir Paul Bloom et Tim P. German, « Two Reasons to Abandon the False Belief Task as a Test of Theory of Mind », Cognition, n77, 2000, p. B25-B31.

24 David Leavens, Kim Bart et William D. Hopkins, «BIZARRE chimpanzees do not represent "the chimpanzee" », Behavioral and Brain Sciences, 33 (2-3), 2010, p. 100-101; ou encore David Leavens, Kim Bart et William D. Hopkins, « The Mismeasure of Ape Social Cognition », Animal Cognition, Volume 22/4, 2019, pp 487-504.
} 
l'homo sapiens sapiens, de la même manière que ce dernier est apparenté avec lui ? Par ailleurs, si le raisonnement ontologique (humain versus non-humain) semble insatisfaisant à maints égards, le raisonnement taxonomique (identifiant les traits propres à l'espèce) l'est tout autant. La critique du premier est devenue commune : le « règne animal » est un ensemble bien disparate, et c'est un truisme de dire qu'il n'y a rien de commun, sur le plan de l'expression des capacités sociales et cognitives, entre un coléoptère et un chien domestique. Cela a pour conséquence de rendre absurdes les formulations du type «les animaux possèdent-ils $\mathrm{X}$ ou $\mathrm{Y}$; sont-ils capables de $\mathrm{X}$ ou $\mathrm{Y}$ » - la catégorie " animaux » ne devenant pertinente que pour penser l'exceptionnalisme humain. La critique du second (le raisonnement taxonomique) est moins répandue. Pourtant, il y a au sein d'une même espèce une grande variation individuelle, surtout entre les individus dits «naïfs » et les individus entrainés en condition expérimentale ${ }^{25}$ (comme le sont la plupart des grands singes dont on met les capacités cognitives à l'épreuve). Aussi, la question de savoir si « les grands singes possèdent $\mathrm{X}$ » ou «les chimpanzés possèdent $\mathrm{Y}$ », ou si «les chiens domestiques sont capables de $\mathrm{Z}$ » est-elle tout aussi inconséquente ${ }^{26}$. C'est là tout le caractère problématique de l'argument comparatiste auquel s'adosse la démarche cognitiviste - et qui met au jour un phénomène de généralisation absolument problématique.

Une autre conséquence directe de cette posture est d'essentialiser les opérations de l'esprit, et les compétences nécessaires à leur mise en œuvre : que ce soit sous forme de "modules » ou encore de compétences au sens chomskyen, il existerait une entité appelée " esprit », et elle existerait indépendamment, presque en amont, des manifestations à travers lesquelles elle est empiriquement identifiée lors des expérimentations ${ }^{27}$. Mais comme les critères définitionnels qui nous servent à évaluer cette entité sont indexés sur ce qui a été mis au jour chez l'humain (et notamment au cours du développement du «petit d'homme »), les dés sont pour ainsi dire pipés dès le départ, car l'objectif est de savoir dans quelle mesure ces "modules» ou cet " esprit » sont proches de ceux que l'on attribue à l'homme ("human-like ») ${ }^{28}$. Et si l'homme est la mesure de toute chose, le chimpanzé ne sera jamais un homme. Retour au sophisme.

Cette essentialisation des concepts d'esprit (mind), d'intention (ou d' " attention conjointe », etc.), devient encore plus problématique quand il s'agit de les éprouver expérimentalement. L'examen de phénomènes cognitifs complexes, requérant de fortes capacités d'abstraction (une «théorie» de l'esprit), s'opère alors par le moyen d'expériences concrètes, en laboratoire ou sur le terrain. Néanmoins, ce sont in fine toujours les comportements (les regards adressés au dominant, les déplacements dans l'espace, la recherche de nourriture, etc.), qui servent d'indices au chercheur pour inférer l'existence d'états mentaux chez l'individu. Cela a il me semble une double conséquence :

a) Les comportements humains seront toujours plus facilement observables, analysables et interprétables que les comportements d'autres espèces animales. Dans le cas d'un protocole comparatif (primate versus enfant), l'observateur humain constate toujours une lisibilité plus grande des actions d'autres humains (même quand ceux-ci sont de

\footnotetext{
${ }^{25}$ Marie Bourjade, «Le pointage controversé des singes : éléments empiriques chez le babouin olive (Papio anubis) », Enfance, n 4 , 2016, p. 375-404.

${ }^{26}$ Pour des critiques répétées, au sein du champ de la cognition sociale, voir Leavens, Bart et Hopkins, idem.

${ }^{27}$ Pour une critique de la «théorie derrière la théorie » de l'esprit (« TToM » pour « Theory Theory of Mind »), voir Alan Costall et Ivan Leudar, «Where is the 'Theory' in Theory of Mind? », Theory Psychology, 2004, vol. 14, p. 623 à 646.

${ }^{28}$ Brian Hare et Michael Tomasello, « Human-Like Social Skills in Dogs? », Trends in Cognitive Sciences, 2005, no 9, p. 439-444.
} 
très jeunes enfants) que d'autres espèces animales avec lesquelles il ne vit pas en contact proximal. C'est tout à fait clair dans les conclusions qui sont tirées, par exemple des travaux de Michael Tomasello, ou encore de ceux de Malinda Carpenter et Josep Call, au sujet de l'attention conjointe. En comparant les manifestations de "checking " (ici l'orientation du regard, le sourire que s'adressent les participants) chez les chimpanzés et les bébés soumis à leurs tests, ils constatent que celles produites par les enfants humains constituent des preuves tangibles à la fois de l'engagement actif des participants dans l'interaction, et de leur orientation mutuelle vers l'objet. Puisque l'enfant rit en regardant sa mère, on déduit qu'il y a bien eu « attention conjointe». Et l'inverse est déduit de l'expérience avec les singes. Or il semble que les comportements des enfants humains sont plus évidemment lisibles, pour les chercheurs, que ceux des chimpanzés. Ce qui manifestement fait défaut, chez les chimpanzés, c'est l'absence de lisibilité spontanée de leurs intentions, qui opère épistémologiquement comme un manque de preuves ("le défi pour les chercheurs qui affirment que les chimpanzés ou les très jeunes enfants s'engagent dans une attention conjointe est donc de fournir une preuve de ce savoir commun $\gg^{29}$ ).

b) C'est là la deuxième conséquence : l'effet «boîte noire» est toujours là, dans la mesure où les expériences mises en œuvre présupposent l'idée qu'il existe quelque chose d'invisible entre un comportement observable et un état mental imputé.

\section{2) Pour une conception pragmatique de l'intentionnalité animale : la cognition comme phénomène ordinaire et situé}

Face à ces spéculations sur une «boite noire» de l'intentionnalité animale, dont on chercherait à éprouver l'existence à travers la plus ou moins grande proximité des facultés de raisonnement des non-humains avec celles des humains, je propose de travailler dans une perspective alternative, partant du caractère non problématique de ces questions dans les interactions ordinaires entre hommes et animaux.

Dans les années 1980, Lawrence Wieder observait déjà l'incroyable différence dans la nature des interactions entre chimpanzés captifs et soigneurs (ou laborantins), selon qu'elles avaient lieu dans le cadre d'une expérimentation, ou en dehors de la pratique scientifique, dans le monde ordinaire ("life-world» dans le texte, inspiré du concept phénoménologique de Lebenswelt). Dans son article intitulé "Behavioristic Operationalism and the Lifeworld: Chimpanzees and Chimpanzee Researchers in Face-to-Face Interaction ${ }^{30}$, il montre comment on dénie aux primates quelque état intentionnel que ce soit dans le cadre de la pratique scientifique, comme la perspective behavioriste le nécessite en principe, mais qu'à côté de ça, les expérimentateurs n'ont aucun mal à les traiter, hors du cadre scientifique, comme des sujets qui postulent réciproquement des «activités mentales (au sens d'Alfred Schutz, dans The Problem of Social Reality) $»^{31}$. Comme le résume Catherine Rémy :

«L. Wieder note la position paradoxale de ces scientifiques qui sont conduits à décrire leurs chimpanzés comme des êtres sans conscience et à s'appuyer sur des

\footnotetext{
${ }^{29}$ Malinda Carpenter et Josep Call, «How Joint Is the Joint Attention of Apes and Human Infants? », In Janet Metcalfe et Herbert Terrace (Eds.), Agency and joint attention, 2013, New York, Oxford University Press, p. 4961.

30 Lawrence Wieder, «Behavioristic Operationalism and the Life-World: Chimpanzees and Chimpanzee Researchers in Face-to-Face Interaction », Sociological Inquiry, 50, 3/4, 1980, p. 75-103.

${ }^{31}$ Lawrence Wieder, ibid., p. 97.
} 
expériences qui soient descriptibles sans référer aux animaux en tant que sujets, alors que, dans le même temps, ils vivent avec ces animaux en les considérant comme des sujets-partenaires et, paradoxalement, comptent sur cette relation intersubjective pour mener à bien leurs recherches descriptibles en termes behaviouristes. $»^{32}$

Il apparaît dès lors intéressant d'aller regarder ce qu'il se passe dans l'ordinaire des interactions entre les hommes et les animaux, pour voir comment cette question de l'intentionnalité animale est mobilisée comme un concept praxique, dans l'expérience vécue et non pas comme l'objet à dévoiler à grand renfort d'expérimentations. Il semble en fait, et c'est désormais le point que j'aimerais illustrer, que l'idée que les animaux au contact desquels nous vivons puissent faire preuve d'intentions plus ou moins complexes, soit non seulement considérée comme acquise, mais surtout serve localement de ressource interactionnelle.

En 2006, Eric Laurier, Ramia Maze et Johan Lundin font paraître un article original intitulé «Putting the Dog Back in the Park: Animal and Human Mind-in-Action » ${ }^{33}$, qui consiste simplement à observer et décrire l'activité de promener son chien dans un parc en Suède. À travers l'analyse des échanges de regards, des postures, ou les références à une indexicalité commune, ils traitent cette activité comme « un accomplissement collectif du chien et de son propriétaire, exhibant méthodiquement des intentions et produisant des objets sociaux $»^{34}$. Ils y déploient un propos radicalement sceptique sur la cognition animale : « ceux qui ont suivi les travaux de Sacks (1992) sur la conversation et l'esprit suspendent l'idée que les individus se comprennent les uns les autres; en revanche ils considèrent qu'il y a des preuves évidentes pour dire que les individus ont des compétences procédurales dans la conduite de leurs actions conjointes ${ }^{35}$.

La question de l'esprit est donc en partie évacuée ; elle l'est en tout cas dans les termes où elle se pose habituellement dans la recherche en cognition sociale, au profit d'une focale sur l'action pratique.

Dans les différentes enquêtes que j'ai pu réaliser depuis dix ans ${ }^{36}, \mathrm{j}$ 'ai eu l'occasion de m'intéresser à la manière dont les participants humains à une interaction interspécifique attribuent spontanément des états intentionnels relativement complexes aux animaux avec lesquels ils interagissent. Le fait que leurs partenaires puissent éprouver des émotions, manifester des états épistémiques (croyances, ignorance) ou des sentiments moraux est même, bien souvent, une prémisse nécessaire à la réalisation efficace de l'interaction. L'exemple des animaux d'assistance est à ce titre emblématique : pour se laisser guider par un chien-guide, il faut consentir à lui attribuer une forme élaborée de rationalité (agentivité, capacité à l'improvisation et à l'ajustement, raisonnement pratique ad hoc, etc.). À chaque fois, l'intentionnalité animale est soit explicitement thématisée et formulée verbalement (typiquement dans les contextes pédagogiques, en présence d'autres participants humains), soit seulement présupposée, mais rendue visible dans le déroulement de l'action. Dans le

\footnotetext{
${ }^{32}$ Catherine Rémy, «Agir avec l'animal. Pour une approche ethnographique des relations hybrides ", L'Année sociologique, vol. 66, no. 2, 2016, pp. 299-318.

${ }^{33}$ Eric Laurier, Ramia Maze, et Johan Lundin, « Putting the Dog back in the Park: Animal and Human Mind-inAction », Mind, Culture and Activity, 13(1), 2006, p. 2-24.

${ }^{34}$ Idib., p. 2 (je souligne).

${ }^{35}$ Ibid., p. 5.

${ }^{36}$ Entre autres: sur les interactions d'êtres humains avec des chiens domestiques, des animaux d'assistance (chiens, chevaux d'équithérapie) et des animaux sauvages (macaques de Barbarie au Maroc).
} 
premier cas, les formulations des états supposés attribués à l'animal ont lieu ex-post, et peuvent constituer des supports pour la négociation collective (est-ce que le chien a eu peur, a-t-il été agressif, a-t-il fait quelque chose de moralement répréhensible ?). Mais il y a également de nombreux cas où les participants s'adressent verbalement directement à l'animal, mimant ainsi la structure de la conversation ordinaire ${ }^{37}$. Cela s'accompagne exceptionnellement de phénomènes de ventriloquie (on parle à la place de l'animal, lui attribuant une voix fictive mais supposée), qui montrent à nouveau comment, dans l'économie d'une interaction, il peut être nécessaire de transformer, par le langage, l'animal domestique en être rationnel. Une dernière modalité, sans doute la plus courante, consiste simplement en des ajustements incarnés. Si on prend la peine de les observer attentivement, les réorientations corporelles, les postures, les mouvements dans l'espace, bref la manière dont on s'ajuste aux actions de l'animal avec lequel on interagit, en disent long sur ce que l'on suppose de leurs compétences interactionnelles, et des intentions qu'ils déploient.

Dans ces cas, de telles inférences, simplement présupposées, ne sont pas formulées verbalement, mais elles sont rendues visibles dans l'action (l'exemple des chiens-guide est à ce titre à nouveau édifiant).

De manière significative également, les participants humains semblent n'avoir aucun mal à résoudre les questions pourtant épineuses qui animent les sciences cognitives contemporaines. La possibilité d'une attention conjointe, par exemple, est rarement mise en doute. Ainsi de cette touriste italienne qui, donnant sa dernière cacahuète au macaque de Barbarie sauvage qu'elle était en train de nourrir, met en exergue son geste et l'objet d'attention commun :



Les deux participants se font face. Le singe est assis sur un muret et Monica distribue sa dernière cacahuète en s'adressant directement à lui : « regarde maintenant».
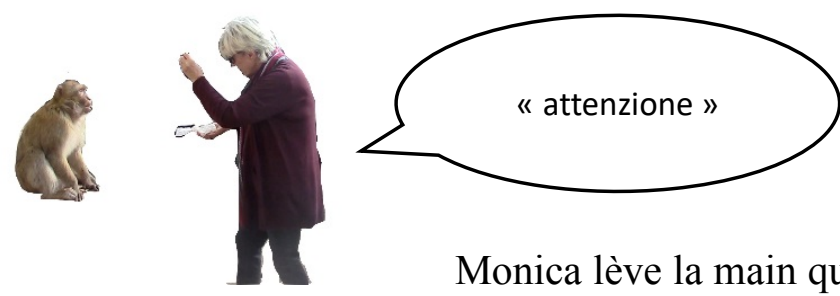

Monica lève la main qui contient la cacahuète en en faisant une référence indexicale. Son geste se double d'un marqueur verbal («attention »). Le singe a le regard orienté vers la main/la cacahuète.

\footnotetext{
37 Pour des cas précis, voir Chloé Mondémé, «Comment parle-t-on aux animaux? Formes et effets pragmatiques de l'adresse aux animaux de compagnie », Langage et Sociétés, 2018, n¹63/1 ; ou encore Chloé Mondémé, "'Y'a un obstacle' : Partage perceptif et construction des savoirs pratiques », in Mondada L. (Ed.). Corps en Interaction : Participation, Spatialité, Mobilité, 2014, Lyon, Ens Editions, p. 191-225.
} 


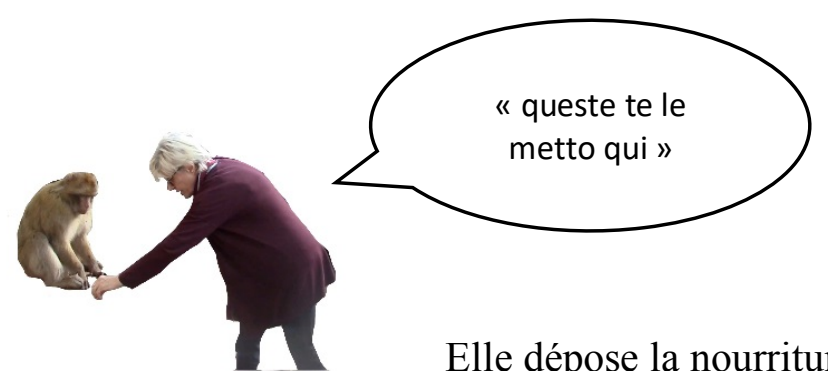

Elle dépose la nourriture devant le singe et glosant son geste («je te la mets ici »). Le singe suit la cacahuète du regard...



Cette scène n'est qu'une illustration, mais on pourrait multiplier les exemples en contexte domestique : désigner un jouet à un chien, concevoir les échanges de regards insistants d'un chat vers sa gamelle comme des formes ordinaires de référence indexicale vers un foyer commun d'attention, etc. Une fois encore, l'idée n'est pas de démontrer que les animaux possèdent des compétences cognitives complexes (la formulation étant, on l'a vu, elle-même extrêmement problématique), mais de voir que les participants aux interactions ordinaires du « monde vécu» semblent, eux, résoudre pratiquement les questions les plus courantes des sciences de l'esprit. Agir avec un animal «comme si » il était doué d'intentionnalité est en ce sens apporter une réponse pratique à la question de l'intentionnalité animale : une réponse qui ne passe pas par la délibération ou l'enquête scientifique, mais qui s'incarne et se rend visible dans l'action.

L'intérêt d'une telle focale sur l'action pratique ordinaire est qu'elle permet de proposer une approche pragmatiste des notions habituellement travaillées par les sciences cognitives. Il n'est plus alors question des capacités cognitives - individuelles ou collectives - des animaux, mais des modalités de l'agir-ensemble interspécifique, à travers les pratiques épistémiques communes (la catégorisation, la (re)connaissance, le raisonnement pratique), qui sont engagées dans l'interaction.

L'opération de respécification, au sens ethnométhodologique, ne consiste donc pas à déplacer le problème des sciences cognitives vers les sciences sociales, mais à élucider les contextes d'usage de ces phénomènes cognitifs, et à montrer comment ils sont mis en œuvre de manière ordinaire.

\footnotetext{
${ }^{38}$ Ces images sont issues de captations vidéo réalisées dans le Moyen Atlas, au cours d'interactions spontanées mettant en présence des macaques de Barbarie sauvages (non captifs) et des touristes locaux (marocains) ou, plus rarement, étrangers. Pour des développements sur la méthodologie naturaliste employée, voir Chloé Mondémé, La socialité interspécifique, 2019, Limoges, Lambert Lucas.
} 
Quelques éléments pour conclure. On a vu comment l'idée d'intentionnalité animale pouvait être mise à l'épreuve dans deux types de contextes : schématiquement, comme un problème scientifique, et comme un phénomène ordinaire (et non-problématique) pour les individus qui vivent au contact d'animaux et interagissent avec eux.

Quand elle est saisie comme une manifestation de l'esprit, l'intentionnalité animale est examinée, en contexte scientifique, au moyen d'outils théoriques (le concept d'esprit, ou celui de théorie de l'esprit), dont la validité est éprouvée empiriquement à l'aide de protocoles expérimentaux. Trois critiques ont été formulées, visant respectivement :

- D'abord, un paradoxe méthodologique : on cherche à examiner un phénomène qui nécessite de mettre en œuvre de complexes capacités communicationnelles (mind reading, attribution d'états mentaux) mais en traitant les éléments du contexte socioaffectif de l'individu testé comme des contaminateurs, ou des biais devant être neutralisés (cf. la critique de Leavens et alii.).

- Ensuite, un problème de logique, qui se fait jour dans un argument circulaire : schématiquement, l'intentionnalité, parce qu'elle n'est observable qu'à travers la lisibilité pratique de comportements familiers (sourires, maitrise du langage, pointage net, etc.), n'est identifiée clairement qu'au sein de l'espèce humaine. Elle est alors de facto construite comme une spécificité humaine. Les animaux n'en possédant par définition pas les caractéristiques, cela fait logiquement persister un doute quant à l'existence d'une forme d'intentionnalité animale.

- Enfin, une attitude théorique, qui repose sur un présupposé essentialiste : l'intentionnalité est quelque chose que l'on peut définir par un ensemble de paramètres, et que l'on pourrait au fond mesurer, au même titre qu'un précipité dans une expérience de chimie.

Face à ces problèmes, j'ai montré l'intérêt d'une approche alternative, nonreprésentationnaliste, et non-individualiste de la cognition ${ }^{39}$. Cette perspective praxéologique est une position théorique et méthodologique qui suppose au moins deux choses : a) que l'on peut aborder les pratiques communicationnelles et signifiantes comme se rendant visibles dans les activités - et non comme l'expression de propriétés incarnées dans les individus, et b) qu'une des manières de rendre visibles ces activités passe par une observation de l'interaction ordinaire, plutôt que par la réalisation de protocoles expérimentaux visant à isoler leurs composants. Cela contribue, on l'espère, à donner aux sciences sociales une place cruciale, et critique, pour penser les phénomènes de l'esprit.

\footnotetext{
${ }^{39}$ Pour un développement, voir Chloé Mondémé, «Extension de la question de "l'ordre social" aux interactions hommes/animaux - une approche ethnométhodologique », L'Année Sociologique, 2016, vol. 66, n², p. 319-350.
} 